\title{
A Small Trivalent Graph of Girth 14
}

\author{
Geoffrey Exoo \\ Department of Mathematics and Computer Science \\ Indiana State University \\ Terre Haute, IN 47809 \\ g-exoo@indstate.edu
}

Submitted: January 5, 2001; Accepted: March 11, 2002.

\begin{abstract}
We construct a graph of order 384, the smallest known trivalent graph of girth 14 .
\end{abstract}

AMS Subject Classifications: 05D25, 05D35

In this note we use a construction technique that can be viewed as a kind of generalized Cayley graph. The vertex set $V$ of such a graph consists of the elements in multiple copies of some finite group $\mathcal{G}$. The action of $\mathcal{G}$ on $V$ is determined by the regular action on each of the copies of $\mathcal{G}$. This induces an action on the edges of the complete graph on $V$. The edge set of the graph we construct is the union of certain of these orbits.

The particular graph we describe is a trivalent graph of girth 14 and order 384. It is constructed as above using a permutation group $\mathcal{G}$ of order 48: the group generated by the following two permutations.

$$
\begin{aligned}
& (1,33,37,6,9,44,8,19,23,18,22,34) \\
& (2,5,40,4,12,16,11,15,27,48,26,30) \\
& (3,25,41,24,14,39,13,38,29,10,28,46) \\
& (7,32,45,31,21,43,20,42,36,17,35,47) \\
& \left(\begin{array}{l}
1,26,20,25,8,12,7,38)(2,21,24,9,11,35,10,22) \\
(3,32,48,19,13,42,4,33)(5,6,28,31,15,18,14,17) \\
(16,34,41,47,30,44,29,43)(23,40,45,39,37,27,36,46)
\end{array}\right.
\end{aligned}
$$

The permutation representation so generated is in fact a regular representation of the underlying abstract group, as can be verified using GAP [4]. 
Now let the vertex set of our graph be $V=\{i \mid 0 \leq i<384\}$ and define the action of $\mathcal{G}$ on $V$ as follows. For each $\sigma \in \mathcal{G}$ define a permutation $\sigma^{*}$ on $V$ by

$$
\sigma^{*}(v)=\sigma(v \bmod 48)+48\lfloor v / 48\rfloor
$$

for all $v \in V$. To construct our graph we choose a set of unordered vertex pairs, $(u, v)$, and for each $\sigma \in \mathcal{G}$ we add the edge $\left(\sigma^{*}(u), \sigma^{*}(v)\right)$ to the graph. To construct the required graph, we use the following 12 vertex pairs, which give us a set of $12 \times 48=576$ edges.

$$
\begin{array}{lllll}
(0,115) & (48,140) & (144,199) & (192,362) \\
(0,126) & (48,173) & (144,261) & (240,289) \\
(0,282) & (48,339) & (192,312) & (288,360)
\end{array}
$$

The construction guarantees that the automorphism group of the graph will have order at least 48; in fact, it has order 96 [3]. Incidently, it is the only trivalent graph of girth 14 and order 384 or less on which $\mathcal{G}$ acts semiregularly. According to [1], this graph is the smallest known trivalent graph of girth 14, improving the previous bound of 406 [2]. An electronic copy of the adjacency list is available at

$$
\text { http://isu.indstate.edu/ge/CAGES/g3.14.384. }
$$

\section{References}

[1] N. Biggs, Constructions for cubic graphs of large girth. Electron. J. Combin., 5 (1998) A1.

[2] M. J. Hoare, On the girth of trivalent Cayley graphs. Graphs and Other Combinatorial Topics (Proceedings of the Third Czeckoslovak Symposium on Graph Theory, Prague 1982), Teubner, Leipzig 1983, pp.109-114.

[3] B. D. McKay, nauty User's Guide (version 1.5). Technical Report TR-CS-87-03 (Department of Computer Science, Australian National University, 1990)

[4] Martin Schönert et al, Groups, Algorithms and Programming, version 4 release 2. Department of Mathematics, University of Western Australia. 